\title{
CYP17 T/C (rs74357) gene polymorphism contributes to polycystic ovary syndrome susceptibility: evidence from a meta-analysis
}

\author{
Xingyan Liu®, Mei Xu, Min Qian and Lindong Yang \\ Department of Obstetrics \& Gynecology, General Hospital of PLA Eastern Theater (Nanjing General Hospital of Nanjing Military Command), Command, \\ Nanjing, China \\ Correspondence should be addressed to X Liu or L Yang: liuxingyan1211@163.com or yanglingdong-123@163.com
}

\begin{abstract}
The cytochrome P450 family 17 (CYP17) is associated with hyperandrogenism in women, and the association between CYP17 gene polymorphism and the risk of polycystic ovary syndrome (PCOS) is not definitive. In order to determine whether the CYP17 T/C (rs74357) gene polymorphism is an exposure risk for PCOS, a comprehensive meta-analysis summarizing 19 studies was performed. The pooled odds ratio (OR) and the corresponding 95\% $\mathrm{Cl}$ were measured under five genetic models, and the stratified analyses by ethnicity, Hardy-Weinberg equilibrium, testosterone levels and BMI in controls were carried out to identify the causes of substantial heterogeneity. The overall results validated that the CYP17 T/C (rs74357) gene polymorphism was significantly associated with PCOS risk in four genetic models. Moreover, the outcomes of subgroup analysis by ethnicity indicated that the frequencies of the $\mathrm{C}$ allele of CYP17 T/C (rs74357) polymorphism were markedly higher in women from Asia than in Caucasians ( $T$ vs C: OR $0.85,95 \% \mathrm{Cl}=0.74-0.99, P<0.05$ ). Therefore, these findings suggested that the CYP17 T/C (rs74357) gene polymorphism played an indispensable part in increasing the susceptibility of PCOS when carrying the $C$ allele, which proposed that the polymorphism of the CYP17 gene may be a predictive factor for the risk of PCOS or an important pathway in PCOS-associated metabolic and hormonal dysregulation.

\author{
Key Words
}

- meta-analysis

- polycystic ovary syndrome

- gene polymorphism

- CYP17
\end{abstract}

\section{Introduction}

Polycystic ovary syndrome (PCOS), characterized by oligoanovulation, hyperandrogenism and polycystic ovarian morphology (1), is a heterogeneous endocrine metabolic disorder among which 3-10\% of women in reproductive age had been affected (2). Hyperandrogenism is regarded as the common characteristic of PCOS, accompanied by luteinizing hormone (LH) hypersecretion, follicle-stimulating hormone (FSH) (3) reduction and insulin resistance (IR). To date, the pathophysiology of hyperandrogenism in PCOS has not been defined even though enormous researches had been performed in this field, which is included as a key point in the diagnostic program. Genetic and environmental factors were recommended by researchers as important contributions to our understanding of PCOS pathogenesis (4).
It is well known that increased androgen was primarily secreted from theca cells in PCOS $(5,6,7)$. A number of studies have reported that the level of androgen in PCOS was augmented with which compared normal women by isolation and cultivation of theca cells $(8,9)$. The excess biosynthesis of androgen in PCOS was attributed to the enhanced expression of steroid-17- $\alpha$-hydroxylase/17,20 lyase (CYP17A1 gene) in theca cells (10).

The cytochrome P450 family 17 (CYP17), which encodes the activity of enzyme 17- $\alpha$-hydroxylase and 17,20 lyase, is characterized as the crucial enzymes on the pathway of androgen biosynthesis (11). In humans, it is coded by CYP17A1 (CYP17 subfamily A member 1 ) gene which is located at chromosome 10q24.32 (12). The

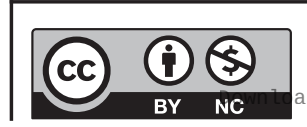

This work is licensed under a Creative Commons Attribution-NonCommercial 4.0 International License. ded from Bioscientifica.com at 04/26/2023 08:14:53AM 
expression of CYP17 could be mainly discovered in the adrenal gland, Leydig cells and theca cells (13). Thus far, a great deal of interest has been shown in single nucleotide polymorphisms (SNPs) in CYP17 gene, a substitution of thymine (T) with cytosine (C) at the 34th bp upstream from the translation initiation point in the promoter region of CYP17 (rs 743572) (14), which hypothesized that additional Sp-1 transcription-binding site was produced and genotyped. Subsequently, the expression of CYP17 was enhanced (15).

More than 100 candidate genes about PCOS associated with metabolism, hormones and inflammation have been explored in numerous researches (16). Genetic trend was identified as the primary etiology, and genome-wide association study (GWAS) widely used in PCOS facilitated us to recognize the pathogenesis in PCOS. The GWAS conducted by Chen on Han Chinese populations had determined the candidate gene loci: DENND1A, RAB5B, INSR, FSHR, LHCGR, YAP1, C9orf3, HMGA2, SUMO1P1, SUOX, TOX3 and THADA $(17,18)$. Afterward, three new risk variants (near PLGRKT, ZBTB16 and MAPRE1) related to PCOS on European women were demonstrated by Day et al. in 2018 (19). Polymorphisms of CYP17 T/C gene have been proved to result in increased synthesis of androgen and a higher risk of the development of several diseases, such as breast cancer (20), prostate cancer (21) and endometriosis (22) et al., which was genotyped in the different studies. Taking into account the disorders of androgen metabolism, CYP17 SNPs have been explained to be involved in various pathophysiologic diseases.

The genes related to hyperandrogenism such as CYP11A1, CYP17A, CYP19, HSD17B5 and HSD17B6 are considered to be potential candidate genes of PCOS. The polymorphism of CYP17 gene had a connection to the risk of PCOS which was discovered in quite a few studies $(20,22$, 23). However, as we know that data from GWAS researches in PCOS on CYP17 polymorphism have not been reported yet and that the current study could be a contribution to the scientific literature. Therefore, our present study was designed to characterize better the association between the CYP17 T/C gene polymorphism and the susceptibility of PCOS.

\section{Materials and methods}

This systematic review and meta-analysis followed the Preferred Reporting Items for Systematic Reviews and Meta-Analyses guidelines (24). As this was a meta-analysis, ethical approval was not required.

\section{Search strategy}

All the available literatures were identified from the following electronic databases: PubMed, Embase, Web of Science, Scopus, Cochrane, and Google Scholar, WanFang Database (Chinese Ministry of Science \& Technology), the Chinese National Knowledge Infrastructure Databases and Chinese Biomedical Database. Studies were searched using the search strategy shown in Fig. 1, from their inception date to November 16, 2020, without restriction on language. The following keywords and combinations were applied: 'polycystic ovary syndrome or PCOS', 'cytochrome p450c17 $\alpha$-hydroxylase or CYP17', 'gene polymorphism'. Besides, the references of the literature were manually screened to identify additional eligible studies.

\section{Study selection}

Eligible studies were involved if they met the following criteria: (i) the study used case-control design; (ii) the correlation between CYP17 T/C gene polymorphism and PCOS was explored in the study; (iii) raw genotype data for each study could be collected; (iv) the diagnosis of PCOS was based on the NICHD standard or the Rotterdam ESHRE/ASRM standard (25).

Studies were excluded from this review if insufficient data (e.g. allele was incomplete) or duplicate data were reported. Studies without a control group were also excluded. When more than one paper was investigated on the same research group, studies without the most recent publication date or the most extensive sample were excluded.

\section{Data extraction}

The literature was screened by two reviewers (Mei Xu and Min Qian), and the data were extracted from all eligible articles separately. Discrepancies were resolved by consensus and arbitration with a third author (Xingyan Liu) if necessary. The following data were collected from included studies: general characteristics (first author, publication year, ethnicity and country), study groups (sample sizes, genotyping method and frequencies of different genotype) and outcome measurement data (serum testosterone levels in total and different genotype, BMI). Furthermore, the Hardy-Weinberg equilibrium (HWE) test was also calculated manually. The characteristics of studies are summarized in Table 1.

\section{Quality assessment}

The overall quality of identified articles was evaluated by two reviewers separately (Xingyan Liu and Lindong Yang) in

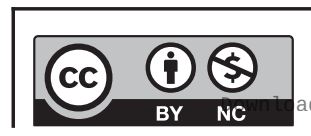

This work is licensed under a Creative Commons Attribution-NonCommercial 4.0 International License. ded from Bioscientifica.com at 04/26/2023 08:14:53AM 

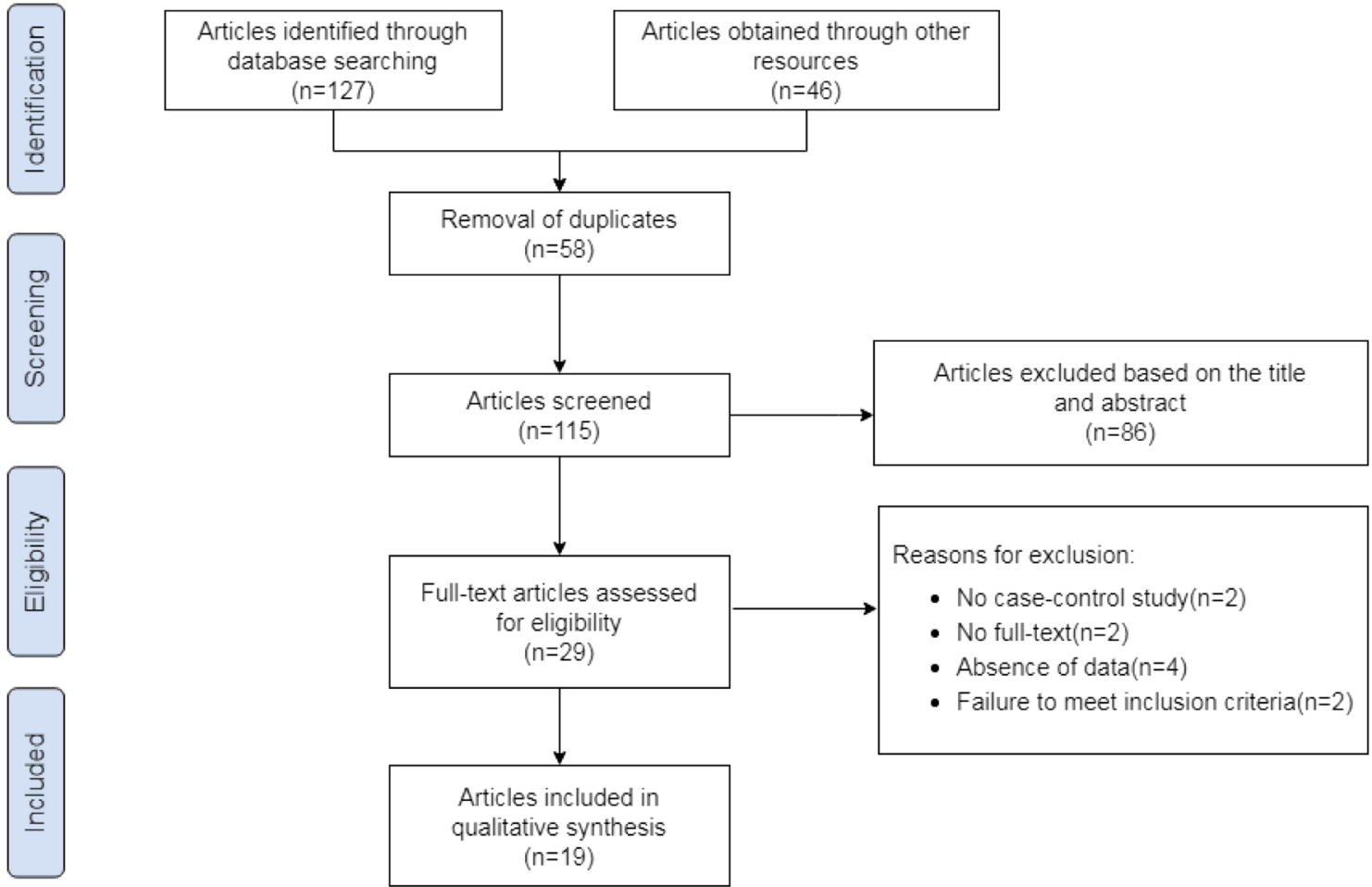

Figure 1

Flowchart for selection of studies.

accordance with the Newcastle Ottawa Quality Assessment Scale (NOS) for case-control studies, in which a star system was used to allow a semi-quantitative assessment for study quality. The NOS was ranged from zero (the worst) to nine stars (the best) in terms of studies' quality.

\section{Statistical analysis}

First of all, HWE was calculated by the chi-square test in the genotype frequencies of controls, from which a $P$ value $<0.05$ was considered as significant departure. Then the pooled odds ratio (OR) and the corresponding 95\% CI were measured to assess the strength of association between CYP17 T/C gene polymorphism and PCOS risk under four genetic models: the allele model (T vs C), the co-dominant model (TT vs CC, TC vs CC, TT vs TC), the dominant model (TT vs TC+CC) and the recessive model (CC vs $\mathrm{TC}+\mathrm{TT}$ ). The significance of ORs was estimated by the Z-test and $P$-value. $P<0.05$ was considered to be indicative of statistically significant. Besides, both the Cochran's Q test and the $\mathrm{I}^{2}$ statistic, which expressed the proportion of variation in this review, were assessed for heterogeneity across studies. A $P$-value $<0.1$ from the Q-test and $\mathrm{I}^{2}>50 \%$ indicated the presence of significant heterogeneity among researches, which the random-effects model was allowed to apply. Otherwise, the fixed-effects model was appropriate. In order to identify the causes of substantial heterogeneity, the stratified analyses by ethnicity, HWE in controls, BMI and testosterone levels were carried out. In addition, sensitivity analyses in which excluded one study at a time were undertaken to assess the stability of the pooled effect. Begg's funnel plot and Egger's test were examined to assess the potential publication bias statistically. All analyses were generated by using STATA 15 (StataCorp, College Station, TX, USA).

\section{Results}

\section{Description of literature}

A total of 115 articles were obtained from the original search after the exclusion of duplicates. The examination of the title and abstract performed on these articles led to the removal of 86 studies and 29 continued to detailed assessment. After screening the full text of these publications, 10 articles were excluded for not meeting the inclusion criteria. Ultimately, 19 eligible case-control studies $(23,26,27,28,29,30,31,32,33,34,35,36,37,38$, $39,40,41,42,43)$ were included in this review in Fig. 1. 


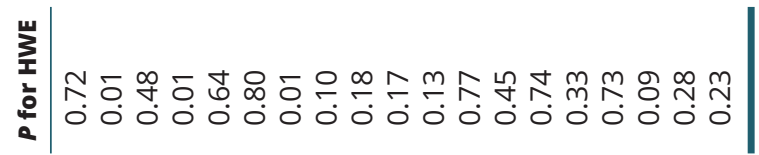

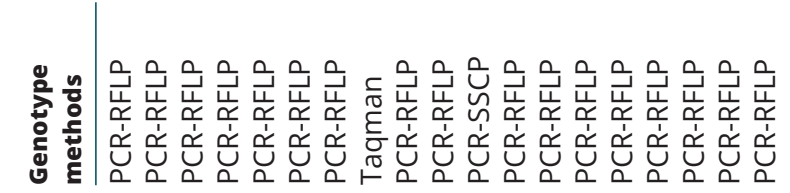

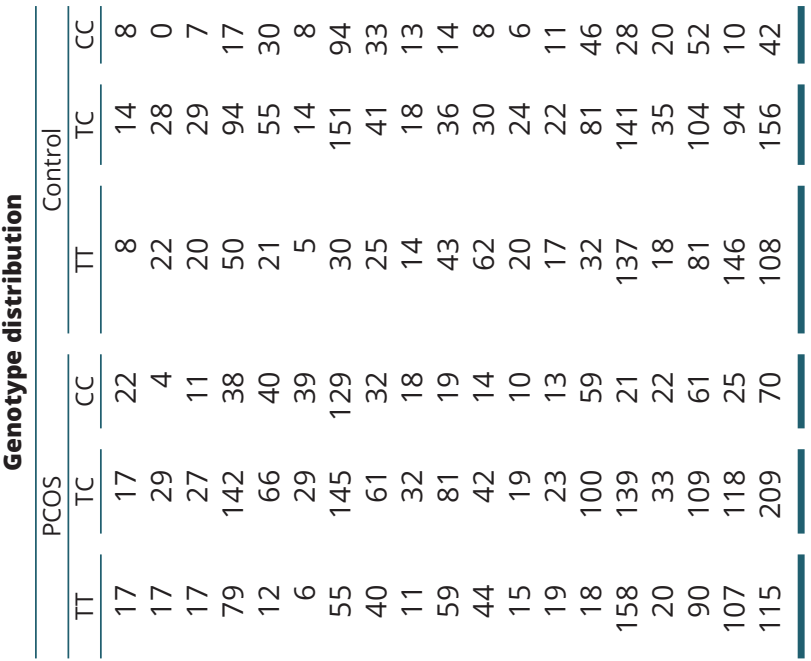

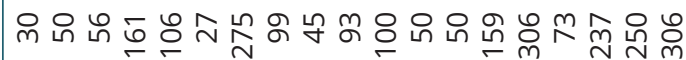

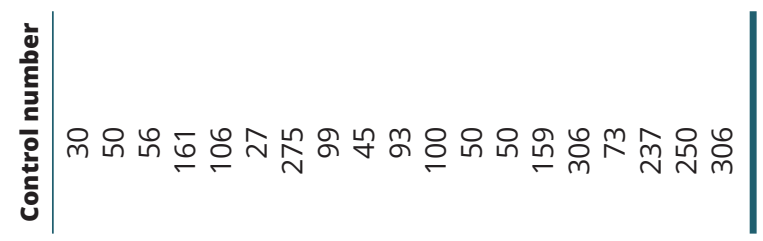

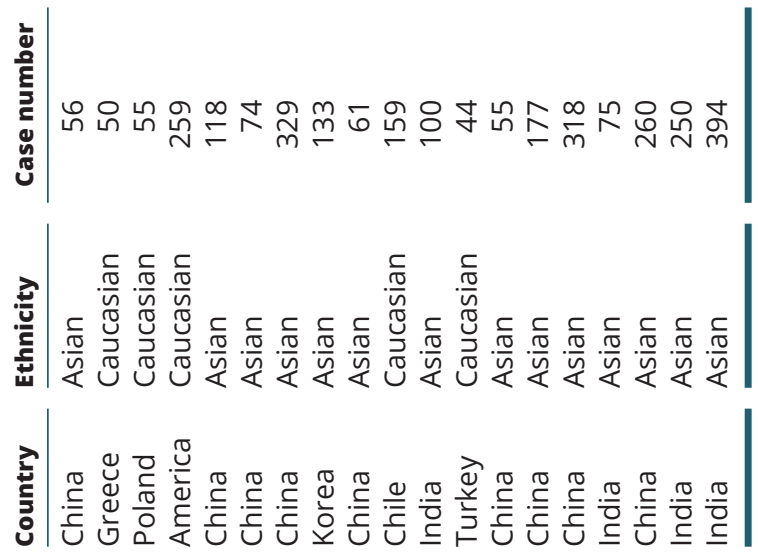

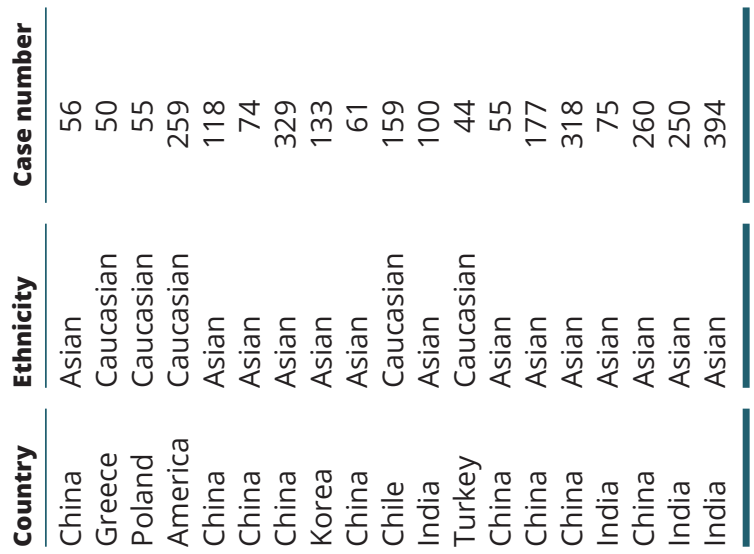

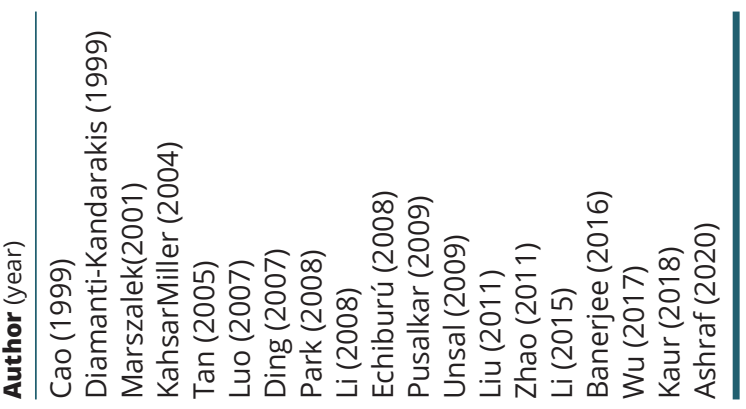

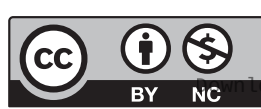


A total of 2967 PCOS patients diagnosed by the Rotterdam or NIH criteria and 2473 non-PCOS participants were involved in our analysis. Fourteen articles were conducted in Asian populations (China, Korea and India), while five articles were carried out in Caucasian populations (Turkey, Chile, Poland, the United States and Greece). Characteristics of included studies are presented in Table 1.

\section{Quality of included reviews}

The NOS employed for case-control studies to evaluate the quality of reviews ranged from 2 to 9 stars, which corresponded to low scores of 7 and high scores of $8-9$. The NOS quality scores were shown in Supplementary Table 1 (see section on supplementary materials given at the end of this article).

\section{Quantitative analysis}

For the overall analysis, our meta-analysis revealed significant association between the CYP17 T/C gene polymorphism and the susceptibility of PCOS in four genetic models: the allele model: ( $\mathrm{T}$ vs $\mathrm{C}$ ) (Fig. 2A) $\mathrm{OR}=0.86,95 \% \mathrm{CI}=0.76-0.97$; the dominant model $(\mathrm{TC}+\mathrm{CC}$ vs TT) (Fig. 2F): $\mathrm{OR}=1.24,95 \% \mathrm{CI}=1.02-1.62)$; the recessive model (TC+TT vs CC) (Fig. 2E): $\mathrm{OR}=0.81$, 95\% CI $=0.69-0.96$; the co-dominant model: (TT vs CC) (Fig. 2B): $\mathrm{OR}=0.72,95 \% \mathrm{CI}=0.56,0.94$; (TC vs CC) (Fig. 2D): $\mathrm{OR}=0.84,95 \% \mathrm{CI}=0.72-0.99$. Stratified analysis was conducted to determine the sources of heterogeneity, which showed that neither ethnicity nor HWE contributed to the observed heterogeneity (Supplementary Figure 1). Nevertheless, the outcome of subgroup analysis by ethnicity indicated that the frequencies of CC genotype and the C allele of the CYP17 $\mathrm{T} / \mathrm{C}$ polymorphism were markedly higher in women from Asia than in Caucasians (T vs C: OR 0.85, 95\% CI $=0.74-$ 0.99; TC vs CC: OR 0.85, 95\%=CI 0.73-1.00; TC+TT vs CC: OR 0.83, 95\% CI=0.69-0.99), while no significant connections were discovered in the Caucasian population under all genetic models in Table 2. Additionally, the effect of the CYP17 polymorphism on testosterone levels was also analyzed, shown in Table 3 . There was a statistically significant difference in PCOS co-dominant model (TC vs CC: $\mathrm{SMD}=-0.21,95 \% \mathrm{CI}=-0.34,-0.07$ ), whereas no difference was observed in the others of PCOS. In contrast, significant differences were existed among various genotypes and BMI, which indicated that the risk of CYP17 genotype in PCOS was robustly associated with BMI.

\section{Publication bias and sensitivity analyses}

Two models were discovered publication bias on the skewed funnel plot (TC+TT vs CC: Begg's test $P=0.025$, Egger's test $P=0.108$; TT vs CC: Egger's test $P=0.041$, Begg's test $P=0.069$ ) (Table 3 ). Studies reporting a positive association in the recessive model (TC+TT vs CC) seem to be over-represented, while smaller studies reporting negative association may be lost in the published papers. However, no significant result in the homozygous model (TT vs CC: $\mathrm{OR}=0.72,95 \% \mathrm{CI}=0.56,0.94$ ) indicated that TT homozygote had no connection with PCOS risk compared with the CC homozygote, suggesting the assumption was indefinite. Begg's funnel plots were negative for publication bias $(P>0.05)$, which was near to symmetry demonstrating no evidence of publication bias under the other models (Fig. 3E and F). Neither was in Egger's bias test. (TT vs TC: Begg's test $P=0.726$, Egger's test $P=0.547$; T vs C: Begg's test $P=0.294$, Egger's test $P=0.353$; TC $+\mathrm{CC}$ vs TT: Begg's test $P=0.363$, Egger's test $P=0.332$ ) (Table 2 ). The sensitivity analysis suggested that no significant influence was yielded on the overall results, which implied the effect of individual study on the overall analysis estimate was stabile (Supplementary Figures 2, 3 and 4).

\section{Discussion}

\section{Principal findings of the study}

Altogether, the higher frequencies of the CC genotype were strongly correlated with women in PCOS than controls, which indicated that the CC genotype and the C allele of the CYP17 T/C (rs74357) gene polymorphism increase PCOS susceptibility. Likewise, the significant correlation of CYP17 polymorphism with BMI, especially in the CC genotype, suggested that the CYP17 T/C (rs74357) polymorphism may be influenced by obesity. Furthermore, the pooled OR in the study by ethnicity indicated that the apparently enhanced frequencies of the CC genotype and C allele occurred only in Asians but not in Caucasians. However, no significant relationship could be discovered among stratified analyses by HWE and testosterone levels. The overall results suggest that not only the gene polymorphism of CYP17 but also obesity could be potential factors to the pathogenesis of PCOS.

PCOS is considered a heterogeneous endocrine syndrome with various genetic, metabolic and environmental abnormalities. Inheritable tendencies have been identified as pathogenesis of PCOS by performed multiple studies during the past two decades. With the

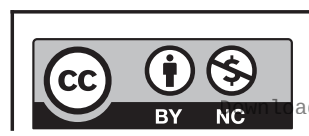

This work is licensed under a Creative Commons Attribution-NonCommercial 4.0 International License. ded from Bioscientifica.com at 04/26/2023 08:14:53AM 
B

A

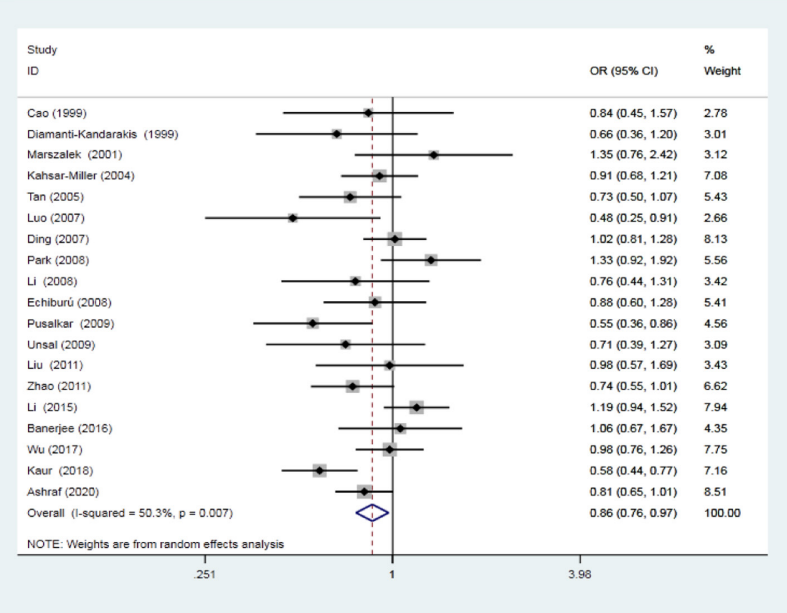

C

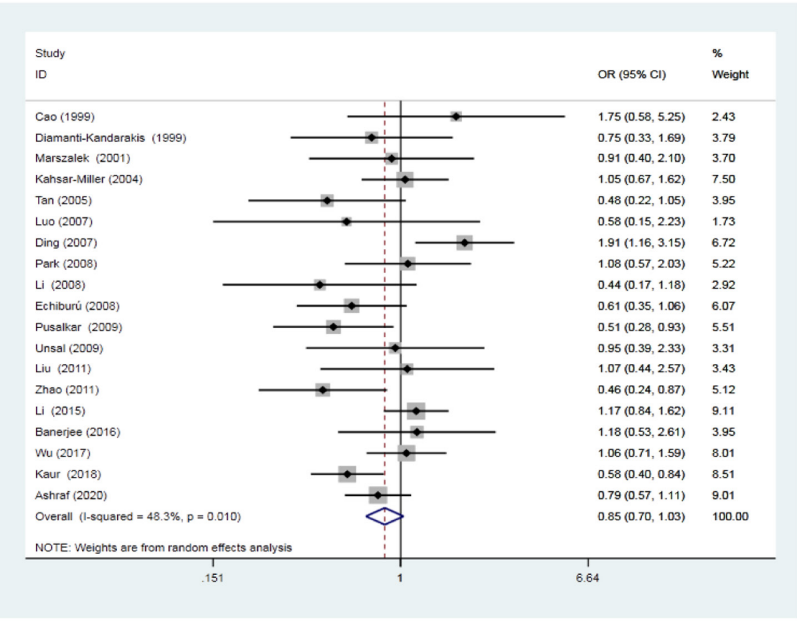

E

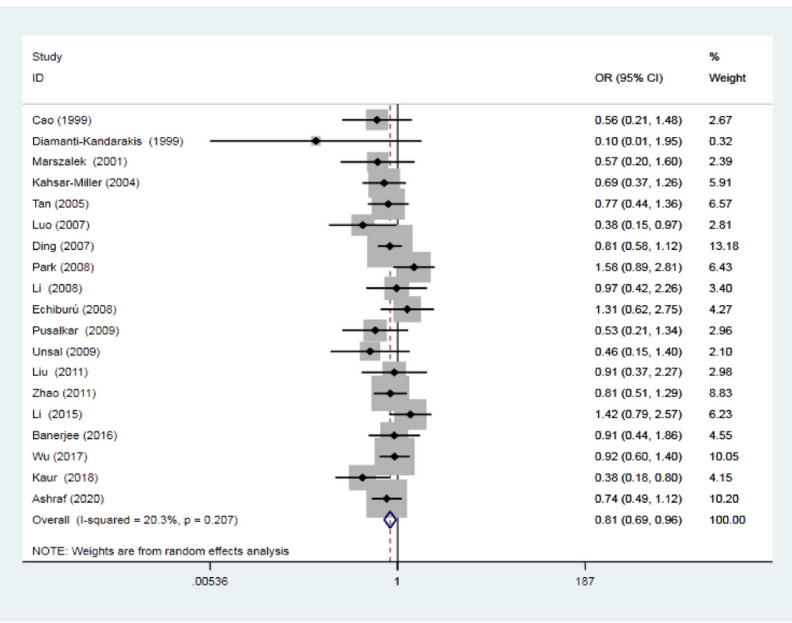

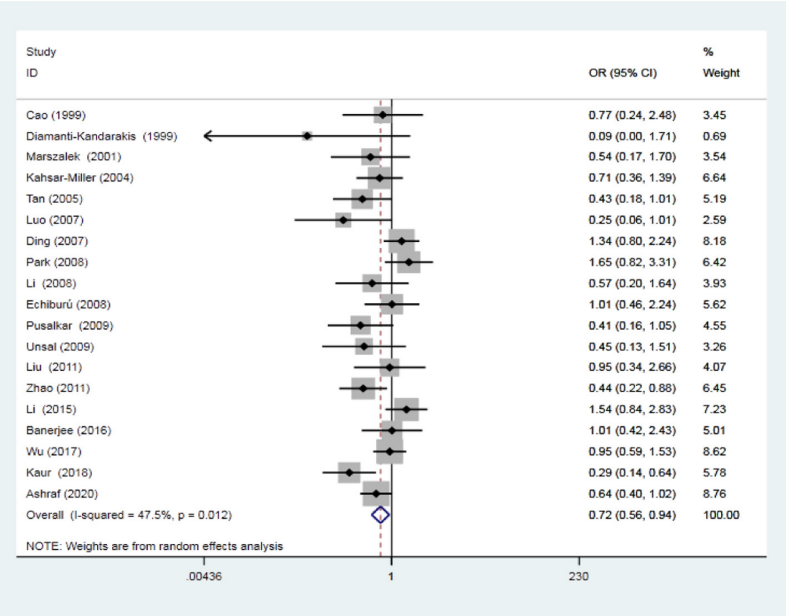

D

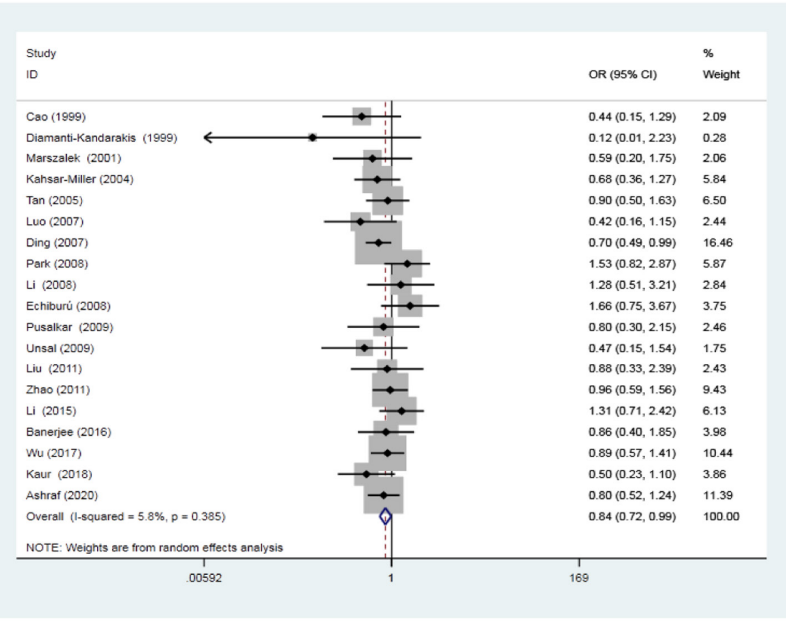

F

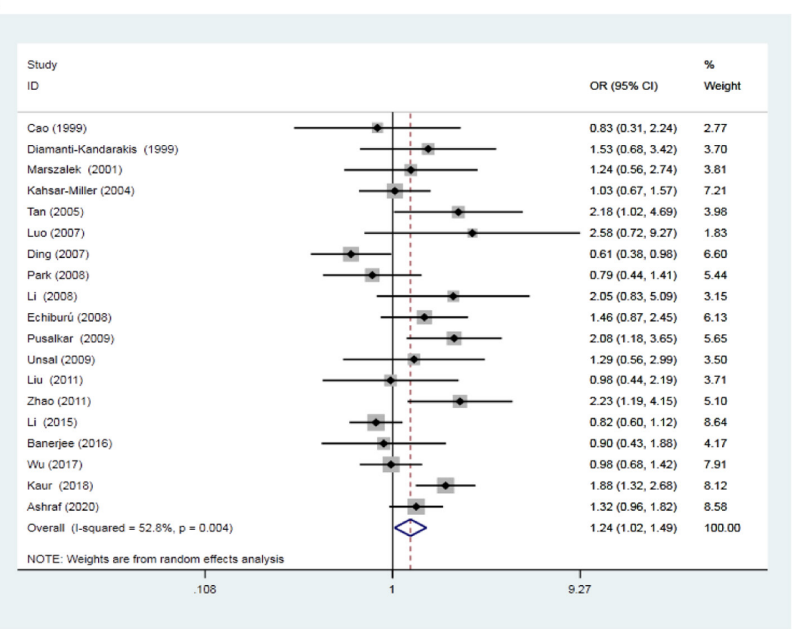

Figure 2

Forest plots of ORs with $95 \%$ Cls for the association between CYP17 T/C polymorphism and PCOS. (A) The allele model (T vs C). (B, C and D) the co-dominant model (TT vs CC, TT vs TC, TC vs CC), (E) the recessive model (TT + TC vs CC) and (F) the dominant model (TC + CC vs TT).

https://ec.bioscientifica.com

https://doi.org/10.1530/EC-21-0327 (c) 2021 The authors Published by Bioscientifica Ltd

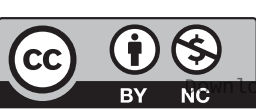

This work is licensed under a Creative Commons Attribution-NonCommercial 4.0 International License.

ded from Bioscientifica.com at $04 / 26 / 2023$ 08:14:53AM 
Table 2 Meta-analysis of the CYP 17 gene polymorphism on PCOS risk.

\begin{tabular}{l} 
Variables \\
\hline Number \\
Allele model (T vs C) \\
OR (95\% CI) \\
$P$-value \\
Co-dominant model \\
TT vs CC \\
OR (95\% CI) \\
$P$-value \\
TT vs TC \\
OR (95\% CI) \\
$P$-value \\
TC vs CC \\
OR (95\% CI) \\
$P$-value \\
Recessive model \\
(TC + TT vs CC) \\
OR (95\% CI) \\
$P$-value \\
Dominant model \\
(TC + CC vs TT) \\
OR ( $95 \% \mathrm{Cl})$ \\
$P$-value \\
\hline
\end{tabular}

\begin{tabular}{c} 
Total \\
\hline 19
\end{tabular}

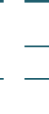

$0.86(0.76,0.97)$

$0.014^{a}$

0.32

$0.85(0.70,1.03)$

0.098

$0.84(0.72,0.99)$

$0.034^{a}$

$0.81(0.69,0.96)$

$0.014^{a}$

$1.24(1.02,1.62)$ $0.029^{a}$
$0.72(0.56,0.94)$

\begin{tabular}{|c|c|}
\hline \multicolumn{2}{|c|}{ Ethnicity } \\
\hline Asian & Caucasian \\
\hline 14 & 5 \\
\hline $0.85(0.74,0.99)$ & $0.89(0.73,1.07)$ \\
\hline $0.033^{a}$ & 0.21 \\
\hline
\end{tabular}

$0.033^{a}$

$0.74(0.54,1.00)$

0.053

$0.68(0.44,1.05)$

0.085

$0.85(0.67,1.09)$

0.198

$0.85(0.64,1.13)$

0.268

$0.85(0.73,1.00)$

$0.049^{a}$

$0.75(0.42,1.32)$

0.314

$0.83(0.69,0.99)$

$0.04^{\mathrm{a}}$

$0.72(0.45,1.15)$

0.165

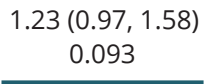

\begin{tabular}{c} 
HWE in con \\
\hline 16 \\
$0.85(0.74,0.98)$
\end{tabular}

$0.025^{a}$

$0.70(0.53,0.92)$

$0.01^{\mathrm{a}}$

$0.79(0.65,0.95)$

$0.015^{a}$

$0.90(0.76,1.07)$

0.237

$0.82(0.68,1.00)$

0.053

$0.76(0.57,1.02)$
0.068

0.108

$0.025^{a}$

aSignificant; $P<0.05=$ significant; $P>0.05=$ non-significant.

HWE, Hardy-Weinberg equilibrium; OR, odds ratio.

advance of technologies in genotype, more than 100 PCOSsusceptible genes have been validated and several of these have determined a significant relationship with PCOS (44). However, the limitation of these researches was a lack of large sample size and conducted on different populations. While a breakthrough was made through numerous explorations, the above problems could be eliminated perfectly by GWAS which provides a benefit for uncovering the associations between genetic variations and diseases.
It represented a significant milestone for PCOS genetics that candidate loci on Han Chinese populations were recognized: DENND1A, RAB5B, INSR, FSHR, LHCGR, YAP1, C9orf3, HMGA2, SUMO1P1, SUOX, TOX3 and THADA (18). McAllister discovered DENND1A.V2, a splice variant of PCOS candidate locus, enhanced CYP17A1 and CYP11A1 gene expression in theca cells and induced the cells to synthesize more androgen (45). Meanwhile, the transcriptional activation of CYP17A1 and CYP11A1 in

Table 3 The association of CYP 17 polymorphism with PCOS according to testosterone and BMI.

\begin{tabular}{|c|c|c|c|c|}
\hline \multirow[b]{2}{*}{ PCOS } & \multicolumn{2}{|c|}{ Testosterone } & \multicolumn{2}{|c|}{ BMI } \\
\hline & & & & \\
\hline \multicolumn{5}{|c|}{ Co-dominant model } \\
\hline TT vs CC & $-0.16(-0.71,0.39)$ & 0.57 & $-0.83(-1.07,-0.58)$ & $<0.0001^{a}$ \\
\hline TT vs TC & $-0.21(-0.34,-0.07)$ & $0.002^{a}$ & $-0.19(-0.36,-0.03)$ & $0.02^{a}$ \\
\hline TC vs CC & $-0.01(-0.14,0.12)$ & 0.88 & $-0.61(-0.84,-0.38)$ & $<0.0001^{a}$ \\
\hline \multicolumn{5}{|l|}{ Recessive model } \\
\hline $\mathrm{TC}+\mathrm{TT}$ vs CC & $-0.04(-0.46,0.39)$ & 0.87 & $-0.72(-0.93,-0.50)$ & $<0.0001^{a}$ \\
\hline \multicolumn{5}{|l|}{ Dominant model } \\
\hline $\mathrm{TC}+\mathrm{CC}$ vs TT & $0.14(-0.13,0.40)$ & 0.32 & $0.33(0.18,0.49)$ & $<0.0001^{a}$ \\
\hline \multicolumn{5}{|l|}{ PCOS vs control } \\
\hline Total & $1.73(1.15,2.31)$ & $<0.0001^{a}$ & $0.58(0.42,0.73)$ & $<0.0001^{a}$ \\
\hline $\mathrm{TT}$ & $0.87(0.35,1.38)$ & $0.0004^{a}$ & $-1.37(-1.67,-1.07)$ & $<0.0001^{a}$ \\
\hline $\mathrm{TC}$ & $1.09(0.95,1.23)$ & $<0.0001^{a}$ & $-0.81(-1.06,-0.55)$ & $<0.0001^{a}$ \\
\hline $\mathrm{CC}$ & $1.03(0.13,1.94)$ & $0.003^{a}$ & $-0.32(-0.66,0.02)$ & 0.06 \\
\hline Total & $1.73(1.15,2.31)$ & $<0.0001^{a}$ & $0.58(0.42,0.73)$ & $<0.0001^{a}$ \\
\hline
\end{tabular}

a Significant; $P<0.05=$ significant; $P>0.05=$ non-significant.

$\mathrm{SMD}$, Std. mean difference.

https://ec.bioscientifica.com

https://doi.org/10.1530/EC-21-0327 (c) 2021 The authors Published by Bioscientifica Ltd

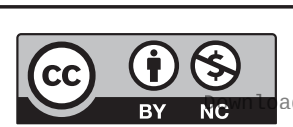

This work is licensed under a Creative Commons Attribution-NonCommercial 4.0 International License. ded from Bioscientifica.com at 04/26/2023 08:14:53AM 
A

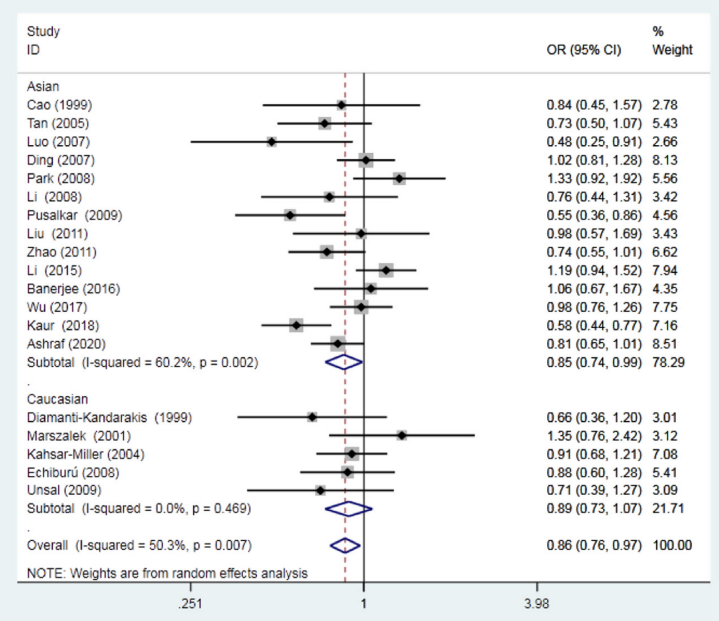

C

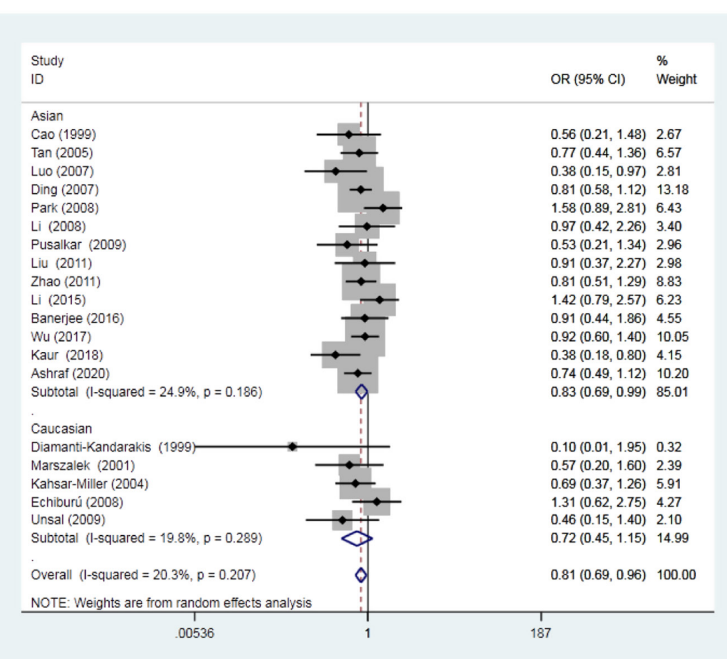

E

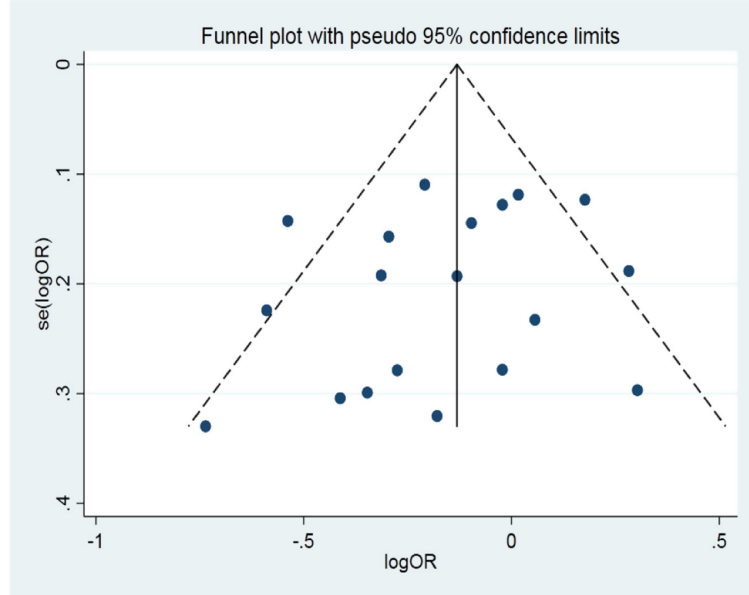

B
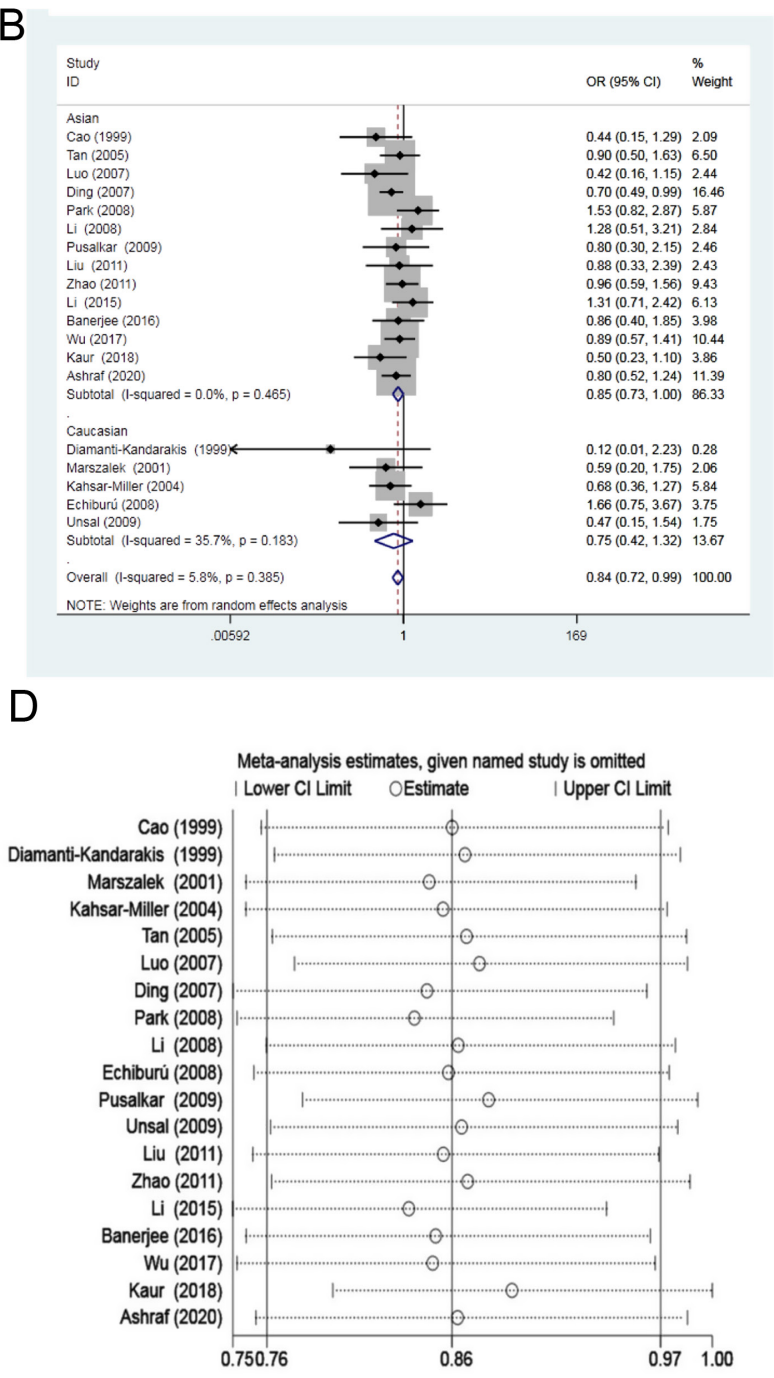

F

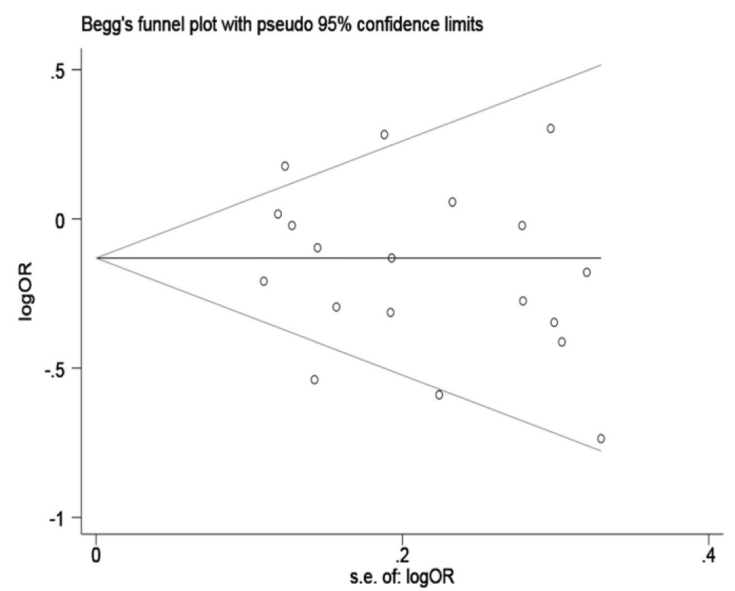

\section{Figure 3}

Statistical analysis for CYP17 T/C polymorphism and risk for PCOS. (A) Subgroup analysis in the allele model (T vs C). (B) Subgroup analysis in the co-dominant model (TC vs CC). (C) Subgroup analysis in the recessive model (TT + TC vs CC). (D) Sensitivity analysis in the allele model (T vs C). (E and F) Funnel plot and Begg's funnel plot with pseudo 95\% confidence limits for publication bias test in the allele model (T vs C).

https://ec.bioscientifica.com https://doi.org/10.1530/EC-21-0327 (c) 2021 The authors Published by Bioscientifica Ltd

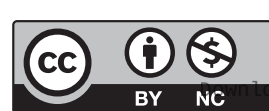

This work is licensed under a Creative Commons Attribution-NonCommercial 4.0 International License. ded from Bioscientifica.com at 04/26/2023 08:14:53AM 
the signaling pathway of DENND1A.V2 was facilitated by RAB5B, another PCOS candidate locus. All of these investigations suggested that CYP17A1 was contained in a steroidogenic signaling cascade and resulted in augmented androgen production. Although researches about the function of CYP17 on PCOS from GWAS have not been conducted, polymorphisms of CYP17 T/C gene have been proved to result in a higher risk of breast cancer (20), prostate cancer (21) and endometriosis (22).

To the best of our knowledge, CYP17 encodes the enzyme 17- $\alpha$-hydroxylase/17,20 lyase (P45017 $\alpha$ ), which is a rate-limiting enzyme in androgen synthesis. DiamantiKandarakis (27) was the first to propose that CYP17 $\mathrm{T} / \mathrm{C}$ gene polymorphism could be responsible for the dysregulation of gene CYP17 expression, which aggravated hyperandrogenemia of PCOS, which was later supported by Pusalkar (39), who described a strong association of CYP17 T/C gene polymorphism with PCOS. In the current study, more frequencies of the polymorphic $\mathrm{C}$ allele and CC genotype were discovered in women with PCOS than in controls, which supported the hypothesis that the significance of the association was found to be more significant compared with controls. Contradictory observations, however, were made in previous studies (23, $27,30)$ where the authors failed to observe any difference in genotype and allele frequencies of the CYP17 T/C gene polymorphism between PCOS and controls.

Consistent with some but not all former studies, stratified analyses by ethnicity were conducted. Ourfindings demonstrated that the $\mathrm{C}$ allele of the CYP17 T/C gene polymorphism was markedly augmented in women from Asia for PCOS according to the allele, recessive, dominant and co-dominant models. This finding is analogous with the study done by Park (38) showing higher expression in the recessive model of CYP17 genotype among PCOS compared with control subjects in a Korean population. However, null associations were observed in Caucasians by Marszalek (37), Echiburú (27) and Ushasi Banerjee (28). Consequently, whether the significant relationship existed in Caucasian women could not be assessed owing to the small number of women in this category, which remains unknown and needs further examination.

CYP17 T/C polymorphism has been respectively discovered to associate with hyperandrogenism and IR in Caucasian women $(27,33)$. Chen had identified insulin receptor (INSR), a strong PCOS candidate locus, played a crucial role in PCOS etiology via IR and metabolic syndrome-related pathways in Chinese women (17). However, no significant correlation was detected between CYP17T/C polymorphism and IR in Thai PCOS women
(46). Thus, it is conceivable that mutations in the INSR gene, like DENND1A gene, possibly converge to upregulate CYP17 expression and androgen biosynthesis through downstream signaling cascades associated with insulin metabolism. Besides, theca cells from PCOS women overexpressed steroidogenic enzymes, particularly CYP17 gene, which explained the steroidogenic disorder of functional ovarian hyperandrogenism in PCOS women (4). Yan Lu considered that the significant role of CYP17 played in glucose metabolic disturbance was mediated by the co-gonadotrophic effects of insulin (47). Consistent with the recent research outcome, the increased BMI associated with CC genotype in our finding provides speculation that CYP17 may affect obesity through a metabolic disorderrelated pathway.

Up to now, quantities of researches on the correlation between CYP17 T/C gene polymorphism and PCOS risk have been carried out. In the previous meta-analysis conducted by Li (48), no connection was discovered between the CYP17 T/C gene polymorphism and PCOS risk in ten case-control studies and some subgroup analyses (by ethnicity and country). Considering the tiny sample size, the discrepancy between the previous analysis and our systemic review was understandable.

\section{Strengths and limitations}

The primary strength of this meta-analysis is the comprehensive search strategy. So far, no preliminary analysis on this issue, which included a larger number of studies in our database than those of previous studies investigating the role of CYP17 T/C gene polymorphism in PCOS, has been as excellent or comprehensive. Moreover, the systematic quality assessment in accordance with the NOS for the quality of study which guaranteed the accuracy and dependability of the conclusions demonstrated that the overall study quality was reasonable. In addition, the sensitivity analysis which was performed to discover influential studies is crucial because these influential studies could lead to reverse results.

However, this review is not without limitations. First, although participants were geographically diverse, more than half of them were categorized as Asian. As shown in the stratified analysis by ethnicity, only five articles were included in the Caucasian population. The metaanalysis conclusions might be attenuated on account of the inadequate sample sizes. Therefore, further studies are warranted to ascertain whether significant connections exist in Caucasians. Secondly, statistically significant heterogeneity was found in the majority of models except

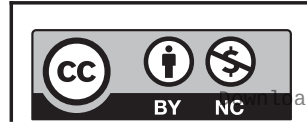

This work is licensed under a Creative Commons Attribution-NonCommercial 4.0 International License. ded from Bioscientifica.com at 04/26/2023 08:14:53AM 
for the recessive model. Unfortunately, conceivable reasons for heterogeneity, such as ethnicity, country, year, HWE in controls, and genotyping methods, could not explain the source of any significant difference by meta-analysis, and we speculated that the discrepancy was caused by gray literature and/or unavailable studies. Thirdly, there was a potential publication bias in the recessive model (TC+TT vs CC) and the co-dominant model (TT vs CC) due to unpublished articles while such a bias could not be assessed in the other models as only studies in English or Chinese included. Last but not leastsince some of the included women showed the presence of Rotterdam criteria I and III, they were diagnosed with PCOS with normal androgen. However, these people were not categorized into the subgroup to discuss the influence of normal androgen levels. So the hypothesis that PCOS women with normal androgens would be affected by the CYP17 polymorphism could not be confirmed, which provides new directions for future research.

\section{Conclusion}

Excess activity of this CYP17 enzyme has been presumed to contribute to increased androgen biosynthesis and secretion in PCOS (49). We hypothesized that CYP17 T/C gene polymorphism was causally correlated with PCOS and the subsequent change of metabolic disorder in PCOS, which indicates the CYP17 T/C gene polymorphism may be potential pharmaceutical targets in fertility treatment and gynecology. In that case, a CYP17 antagonist would be recommended for the prevention of PCOS, as well as for improving symptoms of hyperandrogenemia. The current findings suggest that gene polymorphisms influence the expression and production of CYP17, and the CYP17 T/C (rs74357) gene polymorphism plays an essential role in increasing the susceptibility of PCOS when carrying the $\mathrm{C}$ allele. Close attention should be paid to the diversity of ethnicity because evidence of a relationship in Caucasians was lacking. Despite the convincing connection of CYP17 gene polymorphism to PCOS, the extent to which CYP17 gene polymorphism contributes to metabolic dysfunction in PCOS is unidentified and requires further investigation.

\section{Supplementary materials}

This is linked to the online version of the paper at https://doi.org/10.1530/ EC-21-0327.

\section{Declaration of interest}

The authors declare that there is no conflict of interest that could be perceived as prejudicing the impartiality of this review.

\section{Funding}

This work did not receive any specific grant from funding agencies in the public, commercial, or not-for-profit sectors.

\section{Acknowledgement}

This review was the outcome of an in-house, financially non-supported study. The authors thank the authors of the original studies who responded to the request for data.

\section{References}

1 McCartney CR, Marshall JC \& Marshall JC. Polycystic ovary syndrome. New England Journal of Medicine 2016375 54-64. (https://doi. org/10.1056/NEJMcp1514916)

2 Wolf WM, Wattick RA, Kinkade ON \& Olfert MD. Geographical prevalence of polycystic ovary syndrome as determined by region and race/ethnicity. International Journal of Environmental Research and Public Health 201815 2589. (https://doi.org/10.3390/ijerph15112589)

3 Taylor AE, McCourt B, Martin KA, Anderson EJ, Adams JM, Schoenfeld D \& Hall JE. Determinants of abnormal gonadotropin secretion in clinically defined women with polycystic ovary syndrome. Journal of Clinical Endocrinology and Metabolism 199782 2248-2256. (https://doi.org/10.1210/jcem.82.7.4105)

4 Rosenfield RL \& Ehrmann DA. The pathogenesis of polycystic ovary syndrome (PCOS): the hypothesis of PCOS as functional ovarian hyperandrogenism revisited. Endocrine Reviews 201637 467-520. (https://doi.org/10.1210/er.2015-1104)

5 Jakubowicz DJ \& Nestler JE. 17 $\alpha$-Hydroxyprogesterone responses to leuprolideand serum androgens in obese women with and without polycystic ovary syndromeoffer dietary weight loss. Journal of Clinical Endocrinology and Metabolism 199782 556-560. (https://doi. org/10.1210/jcem.82.2.3753)

6 Gilling-Smith C, Storey H, Rogers V \& Franks S. Evidence for a primary abnormalityin theca cell steroidogenesis in the polycystic ovarian syndrome. Clinical Endocrinology 199747 1158-1165.

7 Nestler JE, Jakubowicz DJ, Vargas AFD, Carlos Brik QN \& Medina F. Insulin stimulates testosterone biosynthesis by human thecalcells from women with polycystic ovary syndrome by activating its own receptor andusing inositolglycan mediators as the signal transduction system. Journal of Clinical Endocrinology and Metabolism $1998 \mathbf{8 3}$ 2001-2005.

8 Nelson VL, Qin KN, Rosenfield RL, Wood JR, Penning TM, Legro RS, Sreauss JF \& Mcallister JM. The biochemical basis for increased testosterone production intheca cells propagated from patients with polycystic ovary syndrome. Journal of Clinical Endocrinology and Metabolism 200186 5925-5933. (https://doi.org/10.1210/ jcem.86.12.8088)

9 Magoffin DA. Ovarian enzyme activities in women with polycystic ovary syndrome. Fertility and Sterility 200686 (Supplement 1) S9-S11. (https://doi.org/10.1016/j.fertnstert.2006.03.015)

10 Wickenheisser JK, Biegler JM, Nelson-Degrave VL, Legro RS, Strauss JF \& McAllister JM. Cholesterol side-chain cleavage gene expression in theca cells: augmented transcriptional regulation and mRNA stability in polycystic ovary syndrome. PLOS ONE 20127 e48963. (https://doi. org/10.1371/journal.pone.0048963)

11 Jahromi MS, Tehrani FR, Noroozzadeh M, Zarkesh M, Ghasemi A \& Zadeh-Vakili A. Elevated expression of steroidogenesis pathway genes; CYP17, GATA6 and StAR in prenatally androgenized rats. Gene 2016 593 167-171. (https://doi.org/10.1016/j.gene.2016.07.067)

12 Nguyen PTT, Conley AJ, Sneyd J, Lee RSF, Soboleva TK \& Shorten PR. The role of enzyme compartmentalization on the regulation of steroid synthesis. Journal of Theoretical Biology 2013332 52-64. (https://doi. $\operatorname{org} / 10.1016 / j . j t b i .2013 .04 .021)$ 
13 Chua AK, Azziz R \& Goodarzi MO. Association study of CYP17 and HSD11B1 in polycystic ovary syndrome utilizing comprehensive gene coverage. Molecular Human Reproduction 201218 320-324. (https://doi. org/10.1093/molehr/gas002)

14 Carey AH, Waterworth D, Patel K, White D, Little J, Novelli P, Franks S $\&$ Williamson R. Polycystic ovaries and premature male pattern baldness are associated with one allele of the steroid metabolism gene CYP17. Human Molecular Genetics 19943 1873-1876. (https:/doi. org/10.1093/hmg/3.10.1873)

15 Carey AH, Chan KL, Short F, White D, Williamson R \& Franks S. Evidence for a single gene effect causing polycystic ovaries and male pattern baldness. Clinical Endocrinology 199338 653-658. (https://doi. org/10.1111/j.1365-2265.1993.tb02150.x)

16 Mitjans M \& Arias B. The genetics of depression: what information can new methodologic approaches provide? Actas Españolas de Psiquiatría 201240 70-83.

17 Chen ZJ, Zhao H, He L, Shi Y, Qin Y, Shi Y, Li Z, You L, Zhao J, Liu J, et al. Genome-wide association study identifies susceptibility loci for polycystic ovary syndrome on chromosome $2 \mathrm{p} 16.3,2 \mathrm{p} 21$ and $9 \mathrm{q} 33.3$. Nature Genetics 201143 55-59. (https://doi.org/10.1038/ng.732)

18 Shi Y, Zhao H, Shi Y, Cao Y, Yang D, Li Z, Zhang B, Liang X, Li T, Chen J, et al. Genome-wide association study identifies eight new risk loci for polycystic ovary syndrome. Nature Genetics 201244 1020-1025. (https://doi.org/10.1038/ng.2384)

19 Day F, Karader T, Jones MR, Meun C, He C, Drong A, Kraft P, Lin N, Huang H, Broer L, et al. Large-scale genome-wide meta-analysis of polycystic ovary syndrome suggests shared genetic architecture for different diagnosis criteria. PLoS Genetics 201814 e1007813. (https:// doi.org/10.1371/journal.pgen.1007813)

20 Chen Y \& Pei J. Factors influencing the association between CYP17 T34C polymorphism and the risk of breast cancer: meta-regression and subgroup analysis. Breast Cancer Research and Treatment 2010122 471-481. (https://doi.org/10.1007/s10549-009-0690-9)

21 Song J, Tao ZH, Liu XY, Gong S \& Gan L. Relationship between CYP17 gene polymorphisms and risk of prostate cancer. Genetics and Molecular Research 201615 15017866. (https://doi.org/10.4238/ gmr.15017866)

22 Cong L, Fu Q \& Gao T. CYP17A1 rs743572 polymorphism might contribute to endometriosis susceptibility: evidences from a casecontrol study. Medicine 201897 e11415. (https://doi.org/10.1097/ MD.0000000000011415)

23 Li L, Gu ZP, Bo QM, Wang D, Yang XS \& Cai GH. Association ofCYP17A1gene-34T/C polymorphism with polycystic ovary syndrome in Han Chinese population. Gynecological Endocrinology 201531 40-43. (https://doi.org/10.3109/09513590.2014.947948)

24 Johansen M \& Thomsen SF. Guidelines for reporting medical research a critical appraisal. International Scholarly Research Notices 20162016 1346026. (https://doi.org/10.1155/2016/1346026)

25 Rotterdam ESHRE/ASRM-Sponsored PCOS Consensus Workshop Group. Revised 2003 consensus on diagnostic criteria and longterm health risks related to polycystic ovary syndrome (PCOS). Human Reproduction 200419 41-47. (https://doi.org/10.1093/humrep/deh098)

26 Kahsarmiller M, Boots LR, Bartolucci A \& Azziz R. Role of a CYP17 polymorphism in the regulation of circulating dehydroepiandrosterone sulfate levels in women with polycystic ovary syndrome. Fertility and Sterility 200482 973-975. (https://doi. org/10.1016/j.fertnstert.2004.05.068)

27 Echiburú B, Pérez-Bravo F, Maliqueo M, Sánchez F, Crisosto N \& SirPetermann T. Polymorphism T $\rightarrow \mathrm{C}$ ( -34 base pairs) of gene CYP17 promoter in women with polycystic ovary syndrome is associated with increased body weight and insulin resistance: a preliminary study. Metabolism: Clinical and Experimental 200857 1765-1771. (https://doi org/10.1016/j.metabol.2008.08.002)

28 Banerjee U, Dasgupta A, Khan A, Ghosh MK, Roy P, Rout JK, Roy P \& Dhara S. A cross-sectional study to assess any possible linkage of C/T polymorphism in CYP17A1 gene with insulin resistance in non-obese women with polycystic ovarian syndrome. Indian Journal of Medical Research 2016143 739-747. (https://doi.org/10.4103/0971-5916.191990)

29 Kaur R, Kaur T \& Kaur A. Genetic association study from North India to analyze association of CYP19A1 and CYP17A1 with polycystic ovary syndrome. Journal of Assisted Reproduction and Genetics 201835 1123-1129. (https://doi.org/10.1007/s10815-018-1162-0)

30 Ashraf S, Rasool SUA, Nabi M, Ganie MA, Jabeen F, Rashid F \& Amin S. CYP17 gene polymorphic sequence variation is associated with hyperandrogenism in Kashmiri women with polycystic ovarian syndrome. Gynecological Endocrinology 202137 230-234. (https://doi. org/10.1080/09513590.2020.1770724)

31 Luo SC \& Zhang YZ. Research on relationship of depressed liverdispersing and liver fire-purging therapy and polycystic ovary syndrome in patients with syndrome of liver excessive heat with the polymorphism of CYP11B2/CYP17 gene. Master's Thesis, Guangzhou Medieal College, 2007

32 Cao YX \& Zhuang GL. Study on the association between polymorphism in the regulatory region of the $17-\alpha$-hydroxylase and 17-20 lyase gene and polycystic ovary syndrome. Progess in Obsterics and Gynecology $19998305-308$.

33 Diamanti-Kandarakis E, Bartzis MI, Zapanti ED, Spina GG, Filandra FA, Tsianateli TC, Bergiele AT \& Kouli CR. Polymorphism T->C (-34 bp) of gene CYP17 promoter in Greek patients with polycystic ovary syndrome. Fertility and Sterility 199971 431-435. (https://doi. org/10.1016/s0015-0282(98)00512-3)

34 Ding LL, Tang R \& Chen ZJ. The association between CYP17 gene polymorphism and high blood testosterone level in Shandong women with polycystic ovary syndrome of han nationality. Master's Thesis, Shandong University, 2007.

35 Li WL \& Cai X. The study of a PolymorPhism in the CYP17 gene and the poly cystic ovary syndrome. Master's Thesis, Xinjiang Medical University, 2008.

36 Liu Z \& Xiao GH. Genetic polymorphism of CYP17, INS and LH $\beta$ genes in patients with polycystic ovary syndrome. Master's Thesis, Guangzhou Medieal College, 2011.

37 Marszalek B, Laciński M, Babych N, Capla E, Biernacka-Lukanty J, Warenik-Szymankiewicz A \& Trzeciak WH. Investigations on the genetic polymorphism in the region of CYP17 gene encoding 5'-UTR in patients with polycystic ovarian syndrome. Gynecological Endocrinology 200115 123-128. (https://doi.org/10.1080/gye.15.2.123.128)

38 Park JM, Lee EJ, Ramakrishna S, Cha DH \& Baek KH. Association study for single nucleotide polymorphisms in the CYP17A1 gene and polycystic ovary syndrome. International Journal of Molecular Medicine 200822 249-254.

39 Pusalkar M, Meherji P, Gokral J, Chinnaraj S \& Maitra A. CYP11A1 and CYP17 promoter polymorphisms associate with hyperandrogenemia in polycystic ovary syndrome. Fertility and Sterility 200992 653-659. (https://doi.org/10.1016/j.fertnstert.2008.07.016)

40 Tan L, Zhang L \& Zhu GJ. Polymorphism of CYP17 gene in patients with polycystic ovary syndrome of Han nationality in China. Journal of Zhengzhou University $2005 \mathbf{4 0} 438-441$.

41 Unsal T, Konac E, Yesilkaya E, Yilmaz A, Bideci A, Ilke Onen H, Cinaz P \& Menevse A. Genetic polymorphisms of FSHR, CYP17, CYP1A1, CAPN10, INSR, SERPINE1 genes in adolescent girls with polycystic ovary syndrome. Journal of Assisted Reproduction and Genetics 200926 205-216. (https://doi.org/10.1007/s10815-009-9308-8)

42 Wu MM, Lu YL, Han L, Liu N \& Wang L. Uyhan and Han nationality patients with polycystic ovary syndrome in Xinjiang: polymorphism of CYP17 gene and its relationship with pathogenesis. Progress in Modern Biomedicine 201717 5305-5308.

43 Zhao CX \& Wang F. Study on the relationship between the polymorphisnm in CYP17 gene and polycystic ovary syndrome in Jimng city. Journal China Health Monthly 20118 25-27.

44 Strauss JF, McAllister JM \& Urbanek M. Persistence pays off for PCOS gene prospectors. Journal of Clinical Endocrinology and Metabolism 2012 97 2286-2288. (https://doi.org/10.1210/jc.2012-2109)

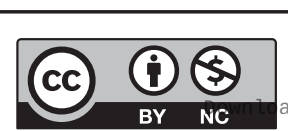

This work is licensed under a Creative Commons Attribution-NonCommercial 4.0 International License. ded from Bioscientifica com at 04/26/2023 08:14:53AM 
45 McAllister JM, Modi B, Miller BA, Biegler J, Bruggeman R, Legro RS $\&$ Strauss JF. Overexpression of a DENND1A isoform produces a polycystic ovary syndrome theca phenotype. PNAS 2014111 E1519-E1527. (https://doi.org/10.1073/pnas.1400574111)

46 Techatraisak K, Chayachinda C, Wongwananuruk T, Dangrat C, Indhavivadhana S, Rattanachaiyanont M \& Thongnoppakhun W. No association between CYP17 -34T/C polymorphism and insulin resistance in Thai polycystic ovary syndrome. Journal of Obstetrics and Gynaecology Research 201541 1412-1417. (https://doi.org/10.1111/jog.12733)

47 Lu Y, Wang E, Chen Y, Zhou B, Zhao Jiejie, Xiang L, Qian Y, Jiang J, Zhao L, Xiong X, et al. Obesity-induced excess of 17-hydroxyprogesterone promotes hyperglycemia through activation of glucocorticoid receptor. Journal of Clinical Investigation 2020130 3791-3804. (https://doi.org/10.1172/JCI134485)

48 Li Y, Liu F, Luo S, Hu H, Li XH \& Li SW. Polymorphism T $\rightarrow$ C of gene CYP17 promoter and polycystic ovary syndrome risk: a meta-analysis. Gene 2012495 16-22. (https://doi.org/10.1016/j. gene.2011.12.048)

49 Yao K, Bian C \& Zhao X. Association of polycystic ovary syndrome with metabolic syndrome and gestational diabetes: aggravated complication of pregnancy. Experimental and Therapeutic Medicine 2017 14 1271-1276. (https://doi.org/10.3892/etm.2017.4642)

Received in final form 18 October 2021

Accepted 17 November 2021

Accepted Manuscript published online 17 November 2021
This work is licensed under a Creative Commons Attribution-NonCommercial 4.0 International License.

ded from Bioscientifica.com at $04 / 26 / 2023$ 08:14:53AM 